\title{
Long non-coding RNA LINC00707 acts as a competing endogenous RNA to enhance cell proliferation in colorectal cancer
}

\author{
HAN WANG ${ }^{1}$, HAIRONG LUAN ${ }^{2}$, TAO ZHAN $^{1}$, XIA LIU $^{2}$, JIE SONG $^{2}$ and HAIBING DAI ${ }^{1,3}$ \\ ${ }^{1}$ First Clinical Medical College, ${ }^{2}$ Basic Medical College, \\ ${ }^{3}$ Key Laboratory of Tumor Prevention and Treatment of Heilongjiang Province, \\ Mudanjiang Medical University, Mudanjiang, Heilongjiang 157011, P.R. China
}

Received April 11, 2019; Accepted November 21, 2019

DOI: $10.3892 /$ etm.2019.8350

\begin{abstract}
Long non-coding RNAs (lncRNAs) have been indicated to serve critical roles in cancer development and progression. Long intergenic non-protein coding RNA 70 (LINC00707) was recently reported to be an oncogene involved in the tumorigenesis of several types of human cancer. However, the clinical role, biological functions and molecular mechanism of LINC00707 in colorectal cancer (CRC) remain unclear. The aim of the present study was to investigate the biological effects and mechanism of LINC00707 in CRC. Reverse transcription-quantitative PCR was used to detect the expression levels of LINC00707 in 65 CRC tissue samples and CRC cell lines (HCT116, HT29 and SW480). Cell Counting Kit-8 and colony formation assays were performed to investigate the effects of LINC00707 on CRC cell proliferation. A dual-luciferase reporter assay was conducted to investigate the mechanisms of LINC00707 in CRC. The upregulation of LINC00707 expression was significantly associated with tumor size, stage and poor survival in patients with CRC. LINC00707 also acted as an independent prognostic factor for CRC. Functional analyses revealed that the knockdown of LINC00707 could inhibit CRC cell proliferation. Furthermore, bioinformatics analysis demonstrated that microRNA (miR)-485-5p could directly bind to LINC00707, which was confirmed by a dual-luciferase reporter assay. In conclusion, the upregulation of LINC00707 is associated with a shorter survival time in patients with CRC. Knockdown of LINC00707 may inhibit the proliferation of CRC cells by binding with miR-485-5p.
\end{abstract}

Correspondence to: Dr Haibing Dai, Key Laboratory of Tumor Prevention and Treatment of Heilongjiang Province, Mudanjiang Medical University, 3 Tongxiang Street, Aimin, Mudanjiang, Heilongjiang 157011, P.R. China

E-mail: haibing_dai@163.com

Key words: colorectal cancer, long non-coding RNAs, LINC00707, miR-485-5p

\section{Introduction}

Colorectal cancer (CRC) is a common human malignancy and the fourth leading cause of cancer-related mortality worldwide (1). CRC is heterogeneous, and its development has been confirmed to be associated with dietary habits, genetic factors and epigenetic changes (2,3). Although an increasing number of oncogenes and tumor suppressor genes have been demonstrated to be involved in the occurrence and development of CRC $(4,5)$, there is still a need to discover new prognostic markers and mechanisms for CRC progression.

Long non-coding RNAs (lncRNAs) are a group of transcripts $>200$ nucleotides long that lack protein-coding capacity (6). IncRNAs have been reported to regulate tumorigenesis and progression in a variety of cancer types (7). A number of studies have confirmed that lncRNAs serve crucial roles in CRC cell proliferation, invasion and drug resistance $(8,9)$. Long intergenic non-protein coding RNA 70 (LINC00707) is located on chromosome 10p14 and has a length of 3,097 bp. LINC00707 has been confirmed to be upregulated in lung adenocarcinoma and hepatocellular carcinoma and act as an oncogene to promote cancer development and progression (10-12). LINC00707 has been demonstrated to promote the proliferation and metastasis of gastric cancer (GC) by interacting with human antigen R (HuR) (13), which indicates that this molecule may also serve an important role in digestive tract tumors. Recent studies have also revealed that LINC00707 is highly expressed in CRC and may promote the progress of CRC by binding to microRNA (miR)-206 $(14,15)$.

In the present study, the expression of LINC00707 in 65 paired CRC and matched non-cancerous tissues (NCTs) was examined. The relationship between LINC00707 and prognosis was also analyzed. Finally, the present study explored the regulatory mechanisms of action of LINC00707 in CRC.

\section{Patients and methods}

Sample collection. A total of $65 \mathrm{CRC}$ tissues and paired adjacent NCTs (located more than $5 \mathrm{~cm}$ from the cancer margin) were obtained from patients undergoing surgery from January 2012 to December 2015 at the First Clinical Medical College of Mudanjiang Medical University (Mudanjiang, China). Inclusion criteria: i) Radical resection of CRC was performed 
in all patients. ii) The patients were pathologically confirmed as suffering from CRC. iii) The clinical and pathological data of the CRC patients were complete. Exclusion criteria: Patients who received preoperative radiotherapy or chemotherapy. The patients were aged between 41 to 72 years (mean age, 60 years). The CRC staging was determined based on the 7 th edition of the American Joint Committee on Cancer and the Union for International Cancer Control staging systems. After tumor resection, the tissue specimens were immediately snap-frozen in liquid nitrogen and then stored at $-80^{\circ} \mathrm{C}$. This study was approved by the Medical Ethics Committees of the Mudanjiang Medical University. All patients signed a written informed consent form.

Cell culture. Human CRC cell lines HCT116 (ATCC ${ }^{\circledR}$ CCL-247 ${ }^{\mathrm{TM}}$ ), HT29 (ATCC ${ }^{\circledR} \mathrm{HTB}-38^{\mathrm{TM}}$ ), and SW480 (ATCC $^{\circledR}$ CCL-228 $^{\mathrm{TM}}$ ) were purchased from the American Type Culture Collection. The human colonic epithelial cell line NCM460 (HTX1841) was obtained from Otwo Biotech, Inc. Cells were cultured in Dulbecco's modified Eagle's medium supplemented with $10 \%$ fetal bovine serum (both from Gibco; Thermo Fisher Scientific, Inc.), $100 \mathrm{U} / \mathrm{ml}$ penicillin and $100 \mu \mathrm{g} / \mathrm{ml}$ streptomycin (both from Beyotime Institute of Biotechnology). The cells were characterized by Genewiz, Inc. using short tandem repeat markers. All cells were confirmed to be mycoplasma-free and were incubated at $37^{\circ} \mathrm{C}$ in a humidified atmosphere containing $5 \% \mathrm{CO}_{2}$.

Reverse transcription-quantitative polymerase chain reaction $(R T-q P C R)$. Total RNA was extracted from frozen tissues and cells using TRIzol ${ }^{\circledR}$ reagent (Invitrogen; Thermo Fisher Scientific, Inc.). Nuclear and cytoplasmic RNA of HT29 and HCT116 cells was extracted using NE-PER nuclear and cytoplasmic extraction reagents (Thermo Fisher Scientific, Inc.) according to the manufacturer's instructions. Complementary DNA (cDNA) was reverse transcribed from $1 \mu \mathrm{g}$ of total RNA using PrimeScript RT Master Mix System (Takara Biotechnology Co., Ltd.). The reverse transcription reaction was performed at $37^{\circ} \mathrm{C}$ for $15 \mathrm{~min}$ and $85^{\circ} \mathrm{C}$ for $5 \mathrm{sec}$. The expression levels of LINC00707, O-GlcNAcylation transferase (OGT) and miR-485-5p were detected by RT-qPCR using the UltraSYBR Mixture (Low ROX) (CWbio Co., Ltd.) in a total volume of $20 \mu \mathrm{l}$ containing cDNA, 2X UltraSYBR Mixture, $0.2 \mu \mathrm{M}$ of each primer and $\mathrm{dd}_{2} \mathrm{O}$. The relative RNA expression was calculated using the $2^{-\Delta \Delta \mathrm{Cq}}$ method (16) and normalized to the internal references ( $\beta$-actin or U6). The primer sequences are listed in Table SI. The PCR amplification procedure included a pre-denaturation step at $95^{\circ} \mathrm{C}$ for $10 \mathrm{~min}$, followed by 40 cycles of $95^{\circ} \mathrm{C}$ for $15 \mathrm{sec}, 59^{\circ} \mathrm{C}$ for $30 \mathrm{sec}$ and $72^{\circ} \mathrm{C}$ for $32 \mathrm{sec}$.

Transfection. HT29 and HCT116 cells were transfected with 50 pmol LINC00707 siRNAs (si-LINC00707), scrambled siRNA (si-blank), miR-485-5p mimics (or NC mimics) or miR-485-5p inhibitor (or NC inhibitor) (Shanghai GenePharma Co., Ltd.) using Lipofectamine ${ }^{\circledR} 2000$ (Invitrogen; Thermo Fisher Scientific, Inc.) according to the manufacturer's instructions. Following transfection for $6 \mathrm{~h}$, the medium was replaced with complete medium. The cells were cultured for $24 \mathrm{~h}$ prior to subsequent experiments.
Table I. Clinicopathological characteristics of 65 patients with CRC.

\begin{tabular}{lrrr}
\hline & \multicolumn{2}{c}{ LINC00707 } & \\
\cline { 2 - 3 } Characteristics & Low & High & P-value \\
\hline Age, years & & & 0.257 \\
$<60$ & 12 & 17 & \\
$\geq 60$ & 20 & 16 & \\
Sex & & & 0.919 \\
Male & 13 & 13 & \\
Female & 19 & 20 & \\
Tumor size, cm & & & $0.017^{\mathrm{a}}$ \\
$<5$ & 23 & 14 & \\
$\geq 5$ & 9 & 19 & \\
Degree of differentiation & & & 0.283 \\
$\quad$ Well and moderate & 28 & 25 & \\
Poor & 4 & 8 & \\
Tumor stage & & & $0.013^{\mathrm{a}}$ \\
I+II & 17 & 13 & \\
III+IV & 15 & 20 & \\
\hline
\end{tabular}

${ }^{\mathrm{a}} \mathrm{P}<0.05$. CRC, colorectal cancer; LINC00707, long intergenic non-protein coding RNA 70.

Cell Counting Kit-8 (CCK-8) and colony formation assays. HT29 and HCT116 cells were seeded at a density of $5 \times 10^{3}$ cells/well onto 96 -well plates and transfected with si-LINC00707 and si-blank. Cell proliferation was assessed for 24, 48, 72 and $96 \mathrm{~h}$ after $10 \mu \mathrm{l} \mathrm{CCK}-8$ reagent (Beyotime Institute of Biotechnology) was added to each well according to the manufacturer's instructions. The optical density values were measured at a wavelength of $450 \mathrm{~nm}$ using a microplate reader (Thermo Fisher Scientific, Inc.). For the colony formation assay, a total of 800 HT29 and HCT116 cells were seeded into 6-well plates and incubated at $37^{\circ} \mathrm{C}$ and $5 \% \mathrm{CO}_{2}$ for 2 weeks. The colonies were fixed with $4 \%$ paraformaldehyde for $15 \mathrm{~min}$ and incubated with trypan blue for $15 \mathrm{~min}$ at room temperature. The number of colonies (more than 50 cells) were counted using light microscopy.

Cell cycle and apoptosis. For cell cycle analysis, HT29 and HCT116 cells with LINC00707-knockdown were stained with PI in the dark for 30 min using a Cell Cycle and Apoptosis Analysis kit (Beyotime Institute of Biotechnology). For cell apoptosis analysis, HT29 and HCT116 cells with LINC00707-knockdown were double stained with Annexin V-FITC and PI at dark for 20 min using an Annexin V-FITC Apoptosis Detection kit (Beyotime Institute of Biotechnology). The cell cycle distribution and the cell apoptosis rates were then determined using a FACSCanto II Flow Cytometer (BD Biosciences). The results were analyzed using ModFit software (version 3.2, Verity Software House, Inc.)

Bioinformatics analysis. The potential binding sites between LINC00707 and miR-485-5p were predicted using the 

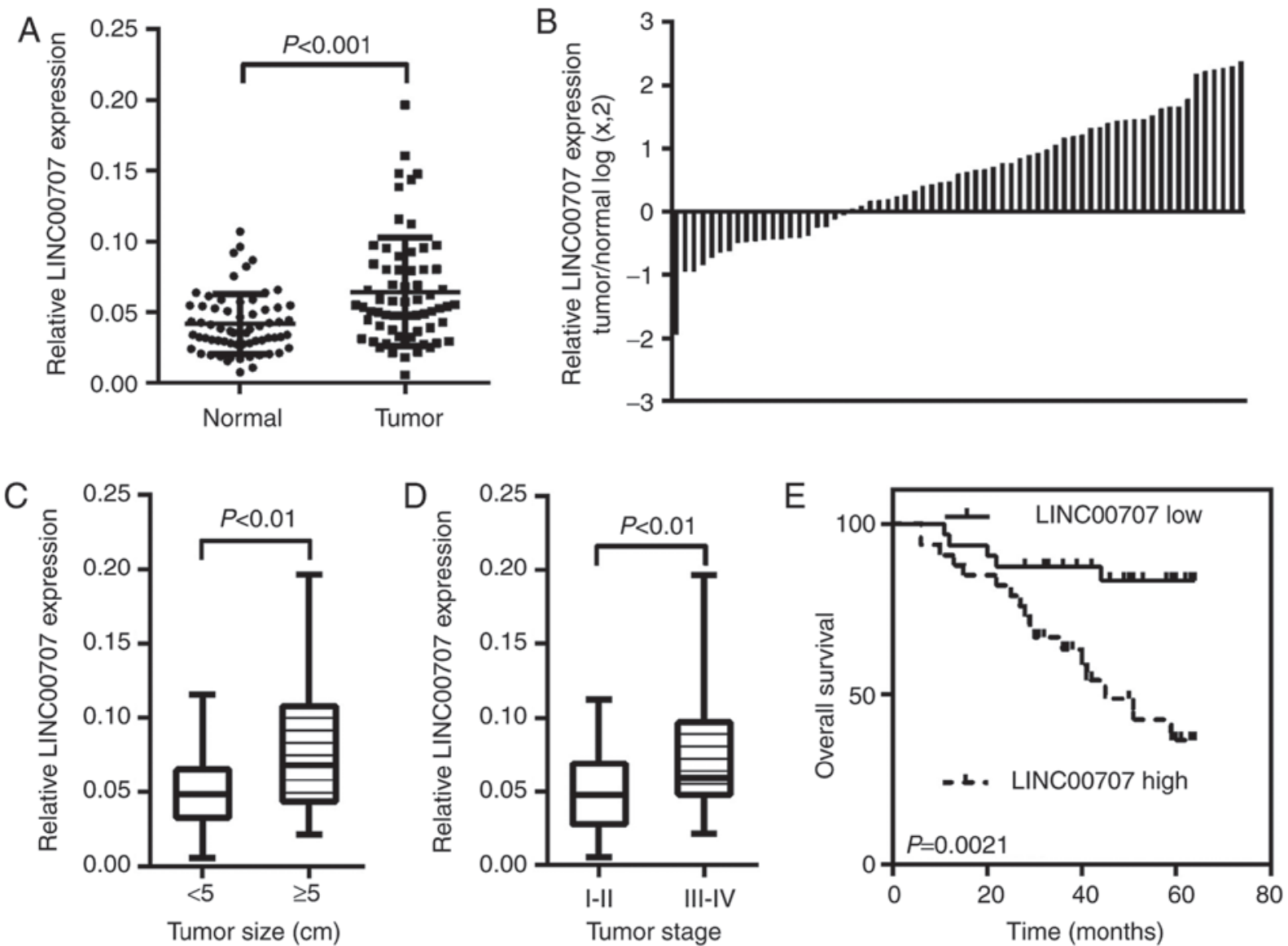

Figure 1. Relative expression of LINC00707 and its association with the prognosis of patients with CRC. (A) The expression levels of LINC00707 in 65 paired CRC tissue samples and their NCTs were quantified by reverse transcription-quantitative PCR. (B) Fold-change in the expression level of LINC00707 in CRC tissues compared with matched NCTs. (C and D) LINC00707 expression in CRC tissues grouped by (C) tumor size $(<5 \mathrm{~cm} \mathrm{vs.} \geq 5 \mathrm{~cm})$ and (D) tumor stage (I+II vs. III+IV). (E) Kaplan-Meier overall survival curves for 65 patients with CRC classified into two groups according to the median expression level of LINC00707 in CRC tissues. LINC00707, long intergenic non-protein coding RNA 70; CRC, colorectal cancer; NCTs, non-cancerous tissues.

starBase v3.0 (http://starbase.sysu.edu.cn/) and RegRNA (http://regrna2.mbc.nctu.edu.tw/index.html) databases.

Vector construction and luciferase reporter assay. The fragments containing the wild-type (Wt) and mutant (Mut) LINC00707 were synthesized and cloned into the luciferase reporter vector pGL3 (Promega Corporation). HT29 and HCT116 cells were co-transfected with LINC00707-Wt or -Mut luciferase reporter vector and miR-485-5p mimics (or NC mimics) using Lipofectamine ${ }^{\circledR} 2000$ (Invitrogen; Thermo Fisher Scientific, Inc.) according to the manufacturer's protocol. The luciferase activities of these cells were measured using the Dual-Luciferase ${ }^{\circledR}$ Reporter Assay System (Promega Corporation) according to the manufacturer's instructions. All firefly luciferase activities were normalized to Renilla luciferase activity.

RNA immunoprecipitation (RIP). Magna RIP kit (EMD Millipore) was used according to the manufacturer's protocol. First, $1 \times 10^{7}$ HT29 and HCT116 cells were washed with ice-cold PBS and lysed using RIP lysis buffer. Then the lysates were centrifuged at $18,000 \times \mathrm{g}$ for $10 \mathrm{~min}$ at $4^{\circ} \mathrm{C}$. Magnetic beads conjugated with anti-argonaute RISC catalytic component 2 (AGO2) (cat. no. 10686-1-AP, $5 \mu \mathrm{g}$ for RIP, ProteinTech Group, Inc.) or anti-IgG (cat. no. PP64B, $5 \mu \mathrm{g}$ for RIP, EMD Millipore) antibodies were used to incubate the cell extract. The cell extract was incubated with gentle agitation overnight at $4^{\circ} \mathrm{C}$. RT-qPCR was conducted to analyze the enrichment of LINC00707 and miR-485-5p.

Western blot analysis. Total protein was extracted from HT29 and HCT116 cells transfected with miR-485-5p inhibitor or si-LINC00707 using RIPA Lysis Buffer (Beyotime Institute of Biotechnology). The protein concentration was measured using an Enhanced BCA Protein Assay kit (Beyotime Institute of Biotechnology). The protein (30 $\mu \mathrm{g}$ per lane) were separated by $10 \%$ SDS-PAGE and transferred to a PVDF membrane. Non-fat milk (5\%) was used to block the membrane at room temperature for $1 \mathrm{~h}$. The membranes were incubated with anti-human OGT (cat. no. 11576-2-AP, 1:2,000; ProteinTech Group, Inc.) and GAPDH (cat. no. AF0006, 1:1,000; Beyotime Institute of Biotechnology) antibodies at $4^{\circ} \mathrm{C}$ overnight. The membranes were subsequently incubated with secondary HRP-labeled goat anti-rabbit IgG (cat. no. A0208, 1:1,000; Beyotime Institute of Biotechnology) and HRP-labeled goat anti-mouse IgG (cat. no. A0216, 1:1,000; Beyotime Institute of Biotechnology) antibodies. The protein levels were detected using an enhanced chemiluminescence system (Pierce; Thermo Fisher Scientific, Inc.). GAPDH was used as the loading control. 
Table II. Univariate and multivariate regression analyses of parameters associated with the prognosis of CRC patients.

\begin{tabular}{|c|c|c|c|c|c|}
\hline \multirow[b]{2}{*}{ Characteristics } & \multirow[b]{2}{*}{ Subset } & \multicolumn{2}{|c|}{ Univariate analysis } & \multicolumn{2}{|c|}{ Multivariate analysis } \\
\hline & & P-value & $\operatorname{HR}(95 \% \mathrm{CI})$ & P-value & HR (95\% CI) \\
\hline Age, years & $\geq 60$ vs. $<60$ & 0.519 & $0.759(0.329-1.753)$ & 0.998 & $1.001(0.401-2.502)$ \\
\hline Sex & Male vs. female & 0.724 & $1.170(0.491-2.790)$ & 0.383 & $1.516(0.595-3.862)$ \\
\hline Tumor size, $\mathrm{cm}$ & $\geq 5$ vs. $<5$ & 0.197 & $1.739(0.750-4.033)$ & 0.441 & $1.530(0.519-4.510)$ \\
\hline Degree of differentiation & $\begin{array}{l}\text { Poor vs. well } \\
\text { and moderate }\end{array}$ & $0.007^{\mathrm{a}}$ & $3.303(1.381-7.898)$ & 0.149 & $2.301(0.741-7.143)$ \\
\hline Tumor stage & III+IV/I+II & $0.001^{\mathrm{a}}$ & $12.042(2.805-51.684)$ & $0.002^{\mathrm{a}}$ & $10.967(2.471-48.671)$ \\
\hline LINC00707 & High/low & $0.005^{\mathrm{a}}$ & $4.255(1.560-11.610)$ & $0.034^{\mathrm{a}}$ & $3.129(1.091-8.968)$ \\
\hline
\end{tabular}

${ }^{\mathrm{a}} \mathrm{P}<0.05$. CRC, colorectal cancer; LINC00707, long intergenic non-protein coding RNA 70.

Statistical analysis. The measurement data were expressed as the mean \pm standard deviation. SPSS 19.0 (IBM Corp.) was used for statistical analysis. The differences between groups were evaluated by two-tailed Student's t-test, $\chi^{2}$ test or one-way ANOVA. One-way ANOVA, followed by Tukey's multiple comparison test, was used to analyze the differences among multiple groups. Survival analysis was performed using the Kaplan-Meier method and the $\log$ rank test. Univariate and multivariate analyses were performed on the basis of a Cox proportional hazard model. Correlations between the LINC00707 and miR-485-5p expression levels, the LINC00707 and OGT expression levels, and between OGT and miR-485-5p expression levels were analyzed using Pearson's correlation coefficient test. $\mathrm{P}<0.05$ was considered to indicate a statistically significant difference.

\section{Results}

LINC00707 is upregulated in CRC tissues. The expression level of LINC00707 was confirmed by RT-qPCR in 65 paired CRC tissues and matched NCTs. The results revealed that LINC00707 expression was significantly increased in tumor tissues compared with that in the corresponding NCTs $(\mathrm{P}<0.001$; Fig. 1A and B). Additionally, the expression of LINC00707 was notably upregulated in patients with large tumor size ( $>5 \mathrm{~cm} ; \mathrm{P}=0.0014$; Fig. $1 \mathrm{C}$ ) and advanced tumor stage (stage III-IV; $\mathrm{P}=0.0028 ;$ Fig. 1D). Collectively, these data suggested that the upregulation of LINC00707 was present in $\mathrm{CRC}$ and may serve a role in CRC progression.

Upregulation of LINC00707 is positively associated with tumor progression and poor prognosis in patients with CRC. To assess the potential association between LINC00707 expression and the clinicopathological characteristics of patients with CRC, the CRC samples were assigned to a high LINC00707-expression group $(n=33$; relative expression level of LINC00707 $\geq$ median value) and a low LINC00707-expression group $(n=32$; relative expression level of LINC00707 < median value). As presented in Table I, high expression of LINC00707 was associated with large tumor size $(\mathrm{P}=0.017)$ and advanced tumor stage $(\mathrm{P}=0.013)$, whereas it was not associated with age, sex or differentiation degree (Table I).
To assess the prognostic value of LINC00707, the effects of LINC00707 expression on the overall survival (OS) of patients with CRC were analyzed. Kaplan-Meier analysis demonstrated that patients with high LINC00707 expression presented a poorer OS compared with those with low LINC00707 expression (log rank=9.453, $\mathrm{P}=0.0021$; Fig. 1E). Univariate analysis demonstrated that the degree of differentiation, tumor stage and LINC00707 expression level were significantly associated with the OS of patients with CRC. Furthermore, multivariate analysis indicated that tumor stage (HR, 10.967; 95\% CI, 2.471-48.671; $\mathrm{P}=0.002)$ and LINC00707 expression (HR, 3.129; 95\% CI, 1.091-8.968; $\mathrm{P}=0.034$ ) were independent prognostic factors for CRC (Table II).

LINC00707 promotes CRC cell proliferation. To further investigate the detailed functional role of LINC00707 in CRC, three CRC cell lines and a normal human intestinal epithelial cell line, NCM460, were subjected to RT-qPCR to examine LINC00707 expression (Fig. 2A). Since high expression of LINC00707 was identified to be associated with large tumor size, the role of LINC00707 in promoting CRC cell proliferation was investigated. LINC00707 expression in HT29 and HCT116 cells was observed to be decreased by siRNAs (Fig. 2B). The results also revealed that the downregulation of LINC00707 significantly decreased cell proliferation and colony formation abilities of CRC cells (Fig. 2C and D). Furthermore, the effect of LINC00707 on the cell cycle of CRC cells was determined by flow cytometry. The results demonstrated that LINC00707 silencing induced cell cycle arrest at the G1 phase compared with the control cells (Figs. 2E and S1). No effect was observed on the apoptotic rates (data not shown). Thus, it was concluded that LINC00707 may promote cell proliferation in CRC.

LINC00707 acts as a competing endogenous RNA (ceRNA) to bind miR-485-5p. To further investigate the underlying mechanism of LINC00707 in CRC, RT-qPCR was performed to detect the localization of LINC00707 in HT29 and HCT116 cells. As presented in Fig. 3A, LINC00707 was mostly distributed in the cytoplasm. Therefore, LINC00707 was hypothesized to participate in post-transcriptional regulation by acting as a ceRNA. The starBase database analysis revealed that LINC00707 could combine with 26 potential microRNAs 
A
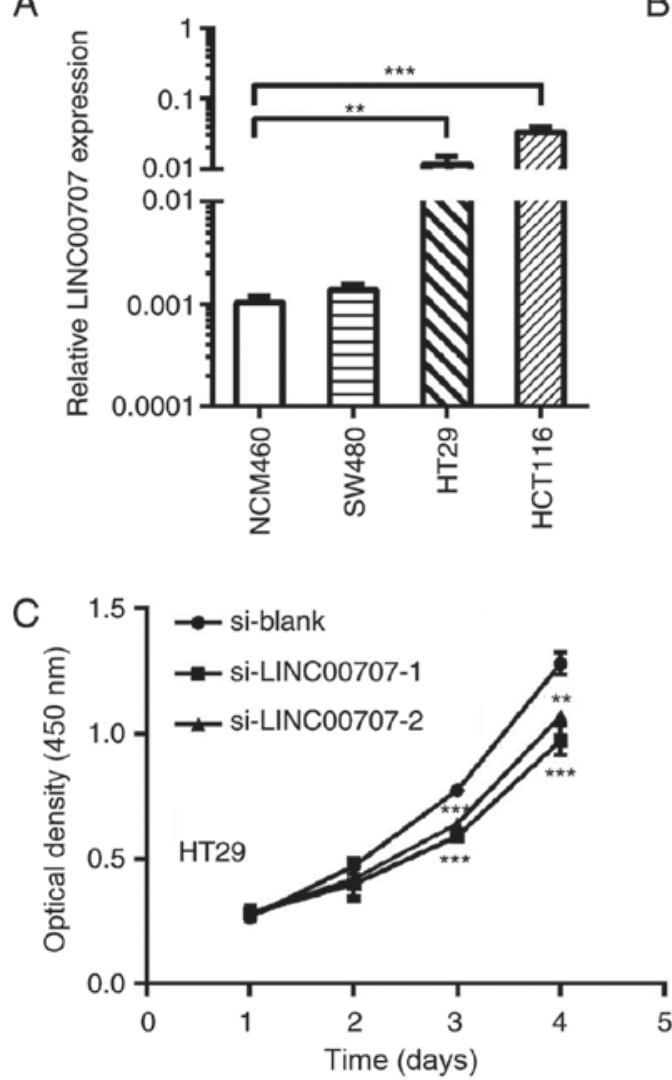

$\mathrm{D}$

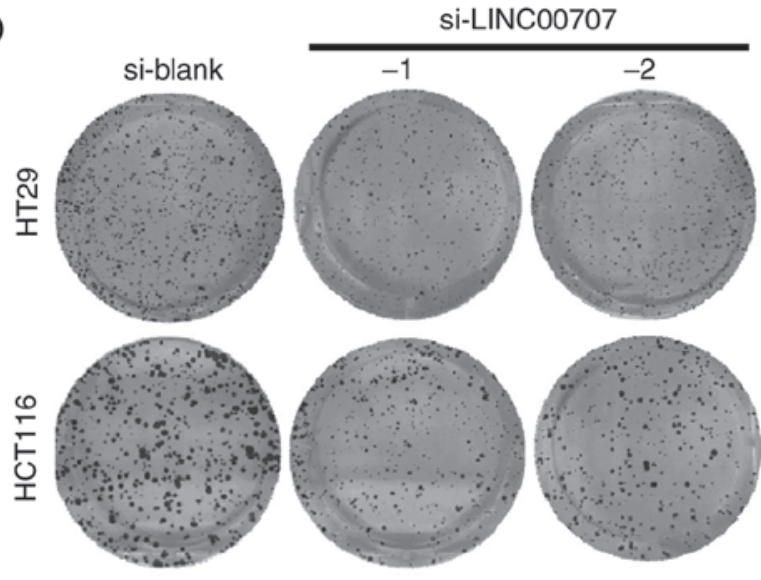

B

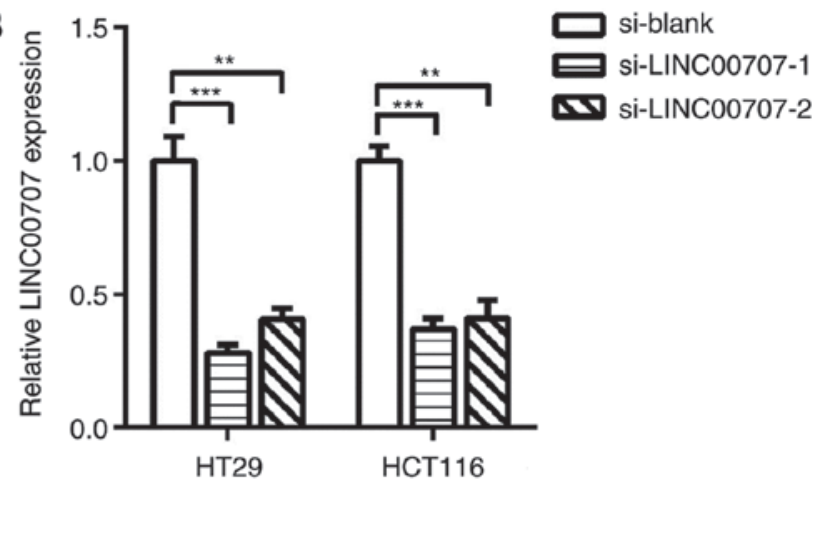

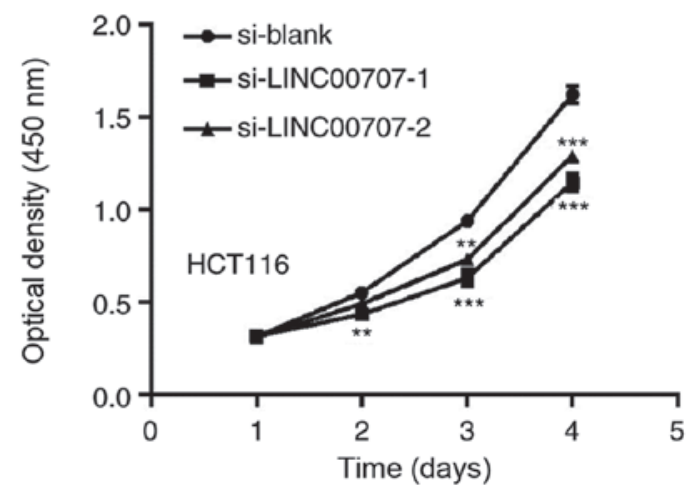
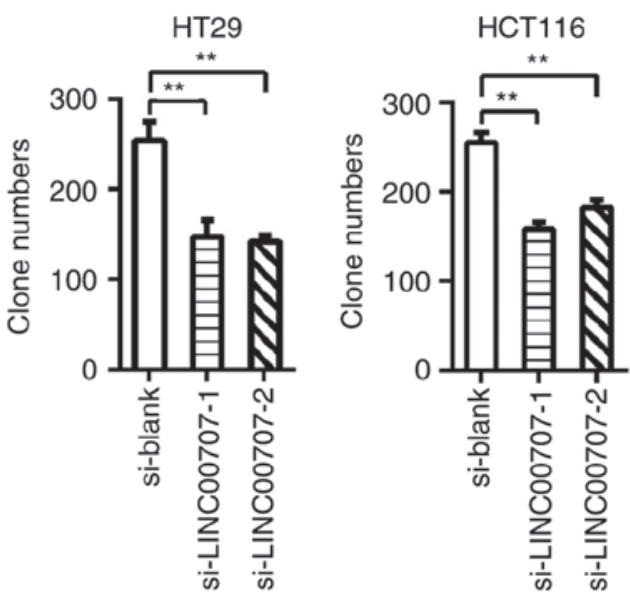

HT29
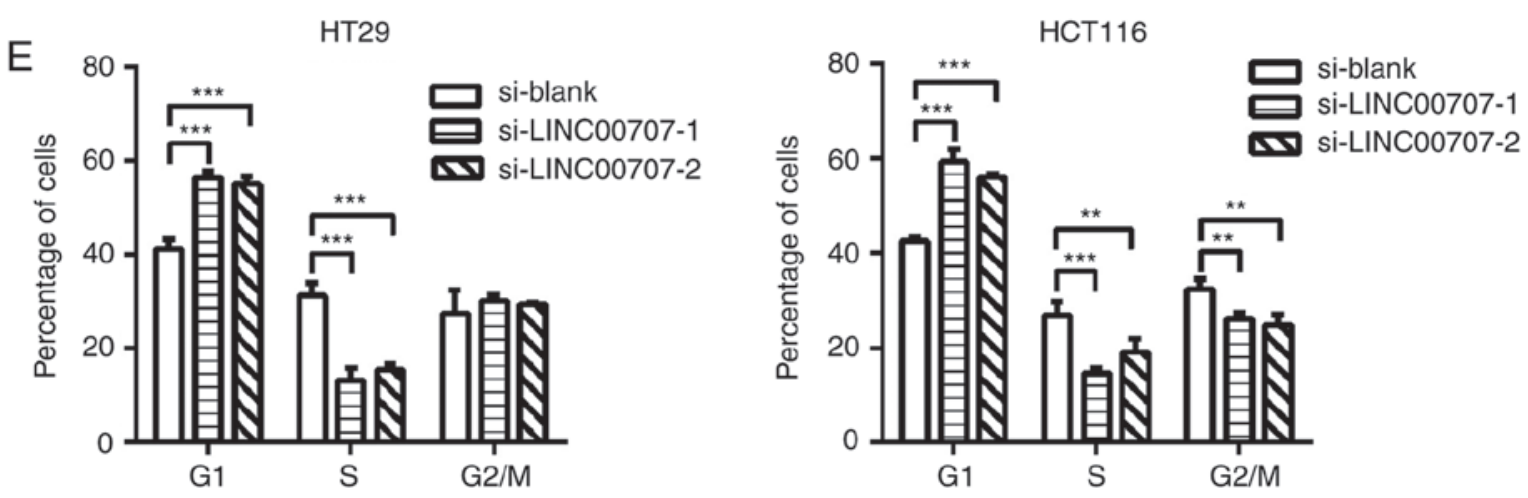

Figure 2. LINC00707 knockdown inhibits CRC cell proliferation. (A) The expression of LINC00707 in CRC cell lines was detected by RT-qPCR (B) LINC00707 was silenced by transfection with siRNAs (si-LINC00707-1 and si-LINC00707-2) in HT29 and HCT116 cells. RT-qPCR was used to detect the transfection efficiency. (C) CCK-8 and (D) colony formation assays were conducted to determine cell proliferation in HT29 and HCT116 cells transfected with si-LINC00707-1 or si-LINC00707-2. (E) Flow cytometry analysis of the cell cycle of HT29 and HCT116 cells transfected with si-LINC00707-1 or si-LINC00707-2. ${ }^{* * *} \mathrm{P}<0.01 ;{ }^{* * * *} \mathrm{P}<0.001$ vs. si-blank. LINC00707, long intergenic non-protein coding RNA 70; CRC, colorectal cancer; RT-qPCR, reverse transcription-quantitative PCR; CCK-8, Cell Counting Kit-8; siRNA, small interfering RNA; si-blank, scrambled siRNA. 
A
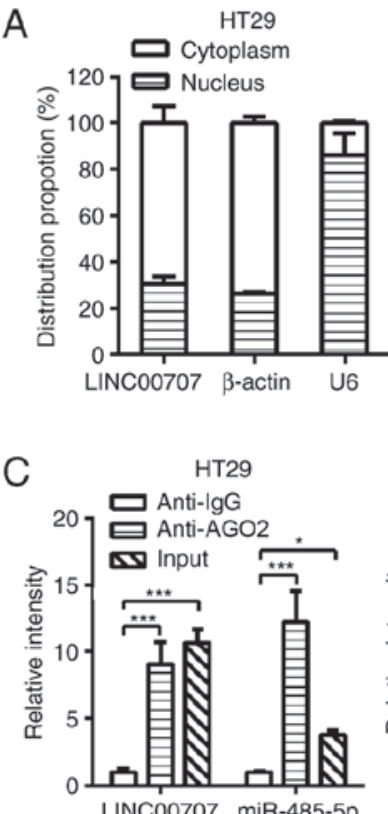
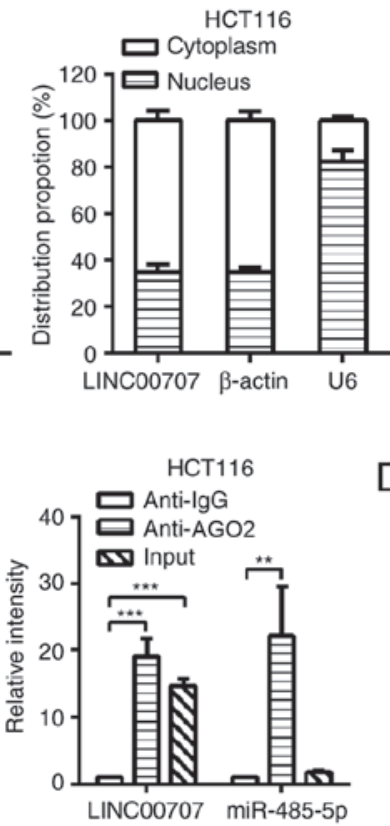

B LINC00707-Wt 5'..CATTTCTCACTAGCCAGCCTCT..

IIIIIIIIII

miR-485-5p 3' CTTAAGTAGTGCCGGTCGGAGA LINC00707-Mut 5'..CATTTCTCACTACGGTCGGAGA..
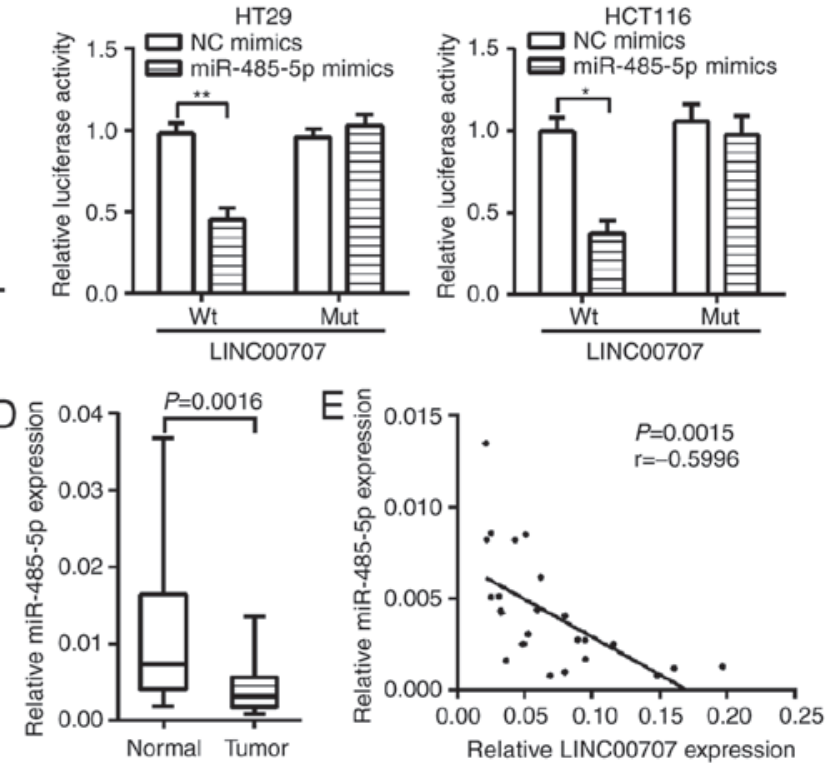

Figure 3. LINC00707 acts as a competing endogenous RNA by sponging miR-485-5p. (A) The localization of LINC00707 in HT29 and HCT116 cells was identified by performing a subcellular fractionation assay. The $\beta$-actin and U6 were used as cytoplasmic and nuclear markers, respectively. (B) The binding site of miR-485-5p to LINC00707 was predicted by bioinformatics analysis and confirmed using a luciferase reporter assay. (C) RNA immunoprecipitation assays were performed in HT29 and HCT116 cells using anti-AGO2 antibody to determine LINC00707 and miR-485-5p RNA enrichment in the immunoprecipitated complex. Anti-IgG was used as the control. (D) The expression level of miR-485-5p was examined in 25 of the 65 pairs CRC and matched non-cancerous tissues. (E) Bivariate correlation analysis of LINC00707 and miR-485-5p expression levels. "P<0.05; " $\mathrm{P}<0.01 ;{ }^{* * * *} \mathrm{P}<0.001$. LINC00707, long intergenic non-protein coding RNA 70; CRC, colorectal cancer; miR, microRNA; AGO2, argonaute RISC catalytic component 2.

(miRNAs), whereas only three potential miRNAs were identified to bind to LINC00707 according to the RegRNA database. The intersection of the predicted data from these two databases showed that miR-485-5p was most likely to combine with LINC00707 (Fig. S2). In addition, a luciferase reporter vector containing LINC00707 was constructed to demonstrate the binding between LINC00707 and miR-485-5p. The results revealed that miR-485-5p mimics could decrease the luciferase activity of LINC00707-Wt, but could not affect the luciferase activity of LINC00707-mut, suggesting that the binding between LINC00707 and miR-485-5p was sequence-dependent (Figs. 3B and S3A). In addition, RIP assay was performed using anti-AGO2 (the key component that associates with miRNAs) in the HT29 and HCT116 extract, and LINC00707 and miR-485-5p were demonstrated to be enriched in the AGO2 compared with anti-IgG immunoprecipitates (Fig. 3C), suggesting that LINC00707 may bind to miR-485-5p through AGO2. Additionally, the expression levels of miR-485-5p in CRC tissues were detected, and the results revealed that miR-485-5p was downregulated in CRC tissues (Fig. 3D). The expression of LINC00707 was also revealed to be inversely correlated with that of miR-485-5p $(\mathrm{P}=0.0015$, r=-0.5996; Fig. 3E).

LINC00707 exerts a tumor-promoting function in CRC by regulating miR-485-5p. To investigate whether LINC00707 promotes cell proliferation in CRC by sponging miR-485-5p, the effect of miR-485-5p inhibition on the proliferation of LINC00707-knockdown HT29 and HCT116 cells was investigated. miR-485-5p inhibition was observed to reverse the effect of LINC00707-knockdown on CRC cell proliferation (Figs. 4A and S3B). A Previous study demonstrated that miR-485-5p inhibits CRC cell proliferation by regulating OGT (17). In the present study, LINC00707 knockdown significantly reduced the endogenous mRNA and protein expression levels of OGT in CRC cells compared with the negative control group (Fig. 4B and C). Silencing of miR-485-5p in LINC00707-knockdown cells restored the expression level of OGT, indicating that LINC00707 may bind miR-485-5p to regulate the expression of OGT. The expression of OGT in 25 of the 65 pairs CRC tissues was also examined, and the results revealed that OGT was notably upregulated in CRC tissues compared with that in NCTs (Fig. 4D). Correlation analysis demonstrated that the expression of OGT was negatively correlated with miR-485-5p levels $(\mathrm{P}=0.0146, \mathrm{r}=-0.4822$; Fig. 4E) and positively correlated with LINC00707 levels $(\mathrm{P}=0.0261$, $\mathrm{r}=0.4442$; Fig. 4F) in CRC tissues.

\section{Discussion}

Currently, emerging evidence has demonstrated that lncRNAs serve increasingly important roles in cancer development and progression. For example, lncRNA-OOC1 has been identified to serve a tumor suppressive role in CRC by destabilizing HuR (18). LINC00659 is a novel oncogenic lncRNA involved in CRC cell growth by regulating the cell cycle (19). LINC00707 is a recently verified lncRNA that has been reported to promote lung adenocarcinoma cell proliferation 

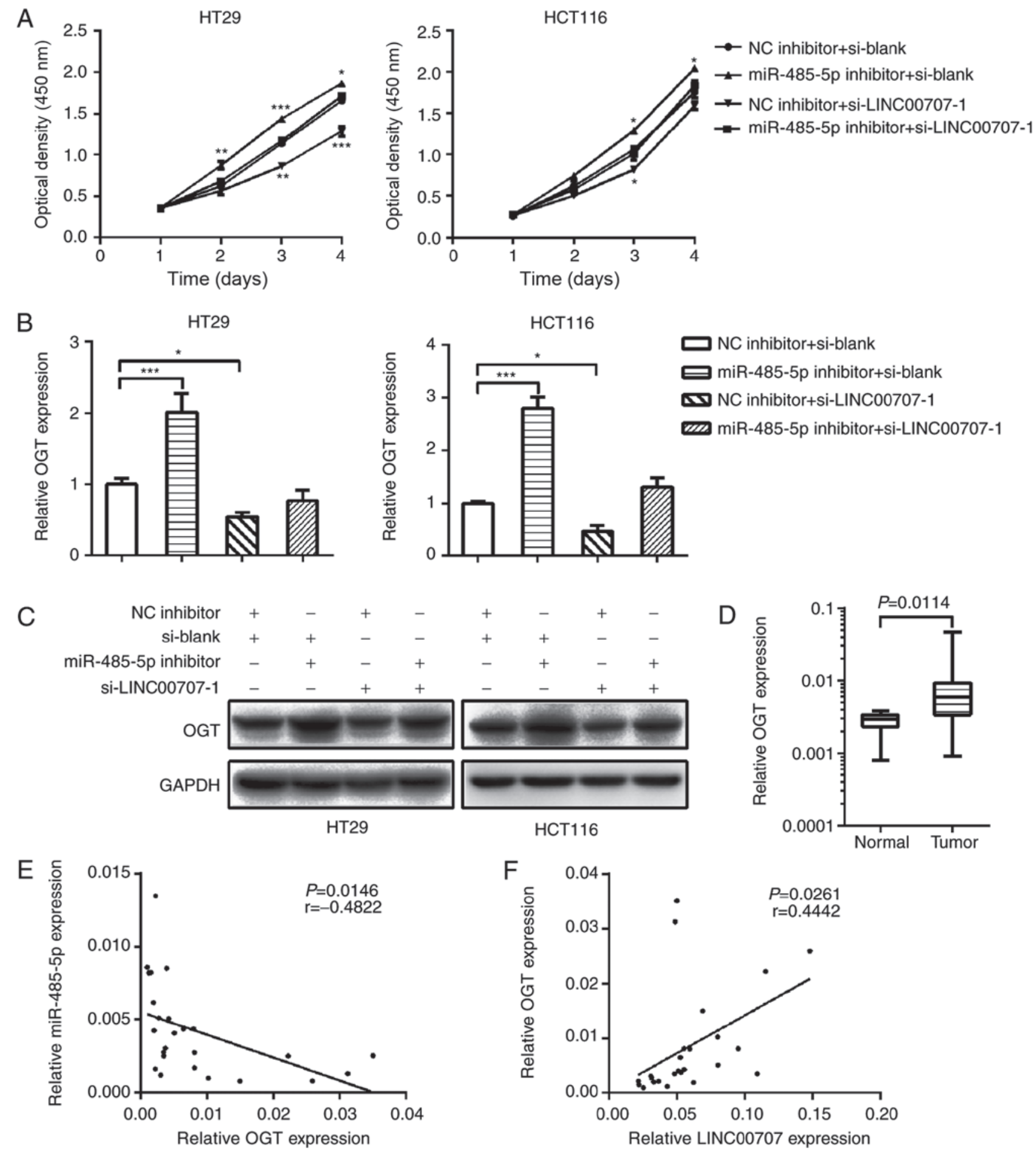

Figure 4. LINC00707 exerts a tumor-promoting function in CRC by regulating miR-485-5p. (A) CCK-8 was used to detect cell proliferation in cells co-transfected with si-LINC00707-1 and a miR-485-5p inhibitor. (B and C) The expression level of OGT in cells co-transfected with si-LINC00707-1 and miR-485-5p inhibitor was examined by reverse transcription-quantitative PCR and western blot analysis. (D) The expression level of OGT was examined in 25 of the 65 pairs CRC and matched non-cancerous tissues. (E) Bivariate correlation analysis of OGT and miR-485-5p expression levels. (F) Bivariate correlation analysis of LINC00707 and OGT expression levels. ${ }^{*} \mathrm{P}<0.05 ;{ }^{* * *} \mathrm{P}<0.01 ;{ }^{* * *} \mathrm{P}<0.001$ vs. NC inhibitor + si-blank. LINC00707, long intergenic non-protein coding RNA 70; CRC, colorectal cancer; CCK-8, Cell Counting Kit-8; OGT, $O$-GlcNAcylation transferase; si, small interfering RNA; si-blank, scrambled small interfering RNA; miR, microRNA; NC, negative control.

and migration by regulating Cdc42 (10). LINC00707 can promote hepatocellular carcinoma progression by activating the extracellular-signal-regulated kinase pathway (11). These studies suggest that LINC00707 is a crucial lncRNA with carcinogenic effects in various types of cancer. In the present study, the significant upregulation of LINC00707 in CRC tissues was observed, which was consistent with the high expression of LINC00707 observed in CRC reported recently by Zhu et al (14) and Shao et al (15). Since the majority of the clinical specimens used in these studies were collected recently and the prognostic information could not be provided, no analysis of the association between LINC00707 and 
prognosis was presented. In the present study, the association between the expression of LINC00707 and the prognosis of patients with CRC was investigated, and the results revealed that high expression of LINC00707 is indicative of poor prognosis in patients with CRC. In addition, the results of the present study demonstrated that LINC00707 could promote CRC cell proliferation, which is consistent with the function of LINC00707 as reported by other studies $(14,15)$.

lncRNAs serve various functions closely related to their cellular localization (20). Cytosolic lncRNAs are frequently involved in post-transcriptional, translational and post-translational regulatory processes by interactions with various proteins or RNA molecules (21). The majority of cytoplasmic lncRNAs serve key roles in the development of human cancers by acting as ceRNAs of miRNAs $(22,23)$. LINC00707 has been demonstrated to induce hepatocellular carcinoma progression through sponging miR-206 and modulating the expression of CDK14 (12). LINC00707 was recently reported to promote osteogenesis by sponging miR-370-3p (24). LINC00707 can act as a molecular sponge for miR-876 to promote the malignant progression of breast cancer (25). LINC00707 mitigates LPS-induced inflammation and apoptosis in PC-12 cells by targeting miR-30a-5p/Neurod 1 (26). It has also been reported that LINC00707 promotes CRC cell proliferation and invasion through sponging miR-206 and regulating the expression of NOTCH3, transmembrane 4 L6 family member 1 and formin-like $2(14,15)$. These studies indicate that LINC00707, as a ceRNA, serves an important role in numerous physiological and pathophysiological processes, especially in the occurrence and development of human tumors. The subcellular localization of LINC00707 was also examined in the present study, and the cytoplasmic location of LINC00707 indicated the potential ceRNA role of LINC00707 in CRC. To date, LINC00707 has been confirmed to bind miR-206, miR-370-3p, miR-876 and miR-30a-5p (12,24-26). As all IncRNAs, LINC00707 can bind multiple miRNAs, as long as they share a miRNA response element. ChIPBase, LncRNAab and starBase were used by Tu et al (12) to identify the interaction between miR-206 and LINC00707 at binding sites 2926-2933. Jia et al (24) demonstrated that miR-370-3p may bind to nucleotides 744-749 of LINC00707 using the LncRNABase and NONCODE databases. Li et al (25) used starBase and LncBase databases to identify two putative binding sites of miR-876 to LINC00707, 593-599 and 604-610. Zhu et al (26) used starBase to identify one putative binding site of miR-30a-5p to LINC00707 at nucleotides 1379-1385. Based on these studies, it may be concluded that there are numerous miRNAs that can bind to different locations of LINC00707. Although it has been reported that LINC00707 can sponge miR-206 in CRC, no explanation has been provided on the selection of miR-206 from a large number of miRNAs that LINC00707 could bind $(14,15)$. Since LINC00707 may bind more miRNAs in CRC, other than miR-206, the intersection of the starBase and RegRNA databases was analyzed in the present study to further predict miRNAs that may be sponged by LINC00707 in CRC. The results revealed that miR-485-5p, as an intersection of these two databases, is likely to bind to LINC00707. Subsequent experiments also confirmed that LINC00707 could bind miR-485-5p and prevent its normal function. miR-485-5p is a tumor suppressor gene in a number of human cancers, such as GC, lung adenocarcinoma, melanoma and breast cancer (27-30). Previous studies have demonstrated the anticarcinogenic role of miR-485-5p in CRC (17,31). The present study demonstrated that LINC00707 could sponge miR-485-5p and inhibit the tumor suppressor effect of miR-485-5p in CRC. The effects of LINC00707 on OGT, a target gene of miR-485-5p, were further examined. The results revealed that the expression of LINC00707 was positively correlated with OGT levels in CRC tissues, indicating that LINC00707 may exert a tumor-promoting function in CRC by regulating miR-485-5p.

A limitation of this study should be noted. The effect of LINC00707 in vivo was not detected. A recent study has reported that LINC00707 promotes CRC growth in vivo (15). The authors used HCT116 cells, which is a type of cells also used in the present study, in a xenograft tumor experiment on nude mice, to knock down the expression of LINC00707. According to the results of Shao et al (15), LINC00707 could promote the proliferation of CRC in vivo.

In conclusion, the results of the present study revealed that LINC00707 was upregulated in CRC tissues and the overexpression of LINC00707 was positively associated with tumor progression and poor prognosis in patients with CRC. In addition, LINC00707 promoted CRC cell proliferation. Further investigation of the underlying mechanism revealed that LINC00707 may function as a ceRNA to sponge miR-485-5p and regulate the expression of the target genes of miR-485-5p. These results indicated that LINC00707/miR-485-5p may promote CRC cell proliferation, providing new insights into $\mathrm{CRC}$ mechanisms and identifying potential therapeutic targets for CRC.

\section{Acknowledgments}

Not applicable.

\section{Funding}

The present study was supported by the Fundamental Research Project for the Colleges and Universities of Heilongjiang Province (grant no. 2018-KYYWFMY-0071).

\section{Availability of data and materials}

All data generated during the present study are included in this published article.

\section{Authors' contributions}

HW, HL and HD designed the study, analyzed the data and wrote the manuscript. HW, TZ, XL and JS performed the experiments. All authors read and approved the final manuscript.

\section{Ethics approval and consent to participate}

The protocol for this study was approved by the Medical Ethics Committee of the Mudanjiang Medical University (Mudanjiang, China). All patients signed a written informed consent form. 


\section{Patient consent for publication}

Not applicable.

\section{Competing interests}

The authors declare that they have no competing interests.

\section{References}

1. Bray F, Ferlay J, Soerjomataram I, Siegel RL, Torre LA and Jemal A: Global cancer statistics 2018: GLOBOCAN estimates of incidence and mortality worldwide for 36 cancers in 185 countries. CA Cancer J Clin 68: 394-424, 2018.

2. Okugawa Y, Grady WM and Goel A: Epigenetic alterations in colorectal cancer: Emerging biomarkers. Gastroenterology 149: 1204-1225, 2015.

3. Fearon ER: Molecular genetics of colorectal cancer. Annu Rev Pathol 6: 479-507, 2011.

4. Sameer AS: Colorectal cancer: Molecular mutations and polymorphisms. Front Oncol 3: 114, 2013.

5. Qi L and Ding Y: Screening of tumor suppressor genes in metastatic colorectal cancer. Biomed Res Int 2017: 2769140, 2017.

6. Necsulea A, Soumillon M, Warnefors M, Liechti A, Daish T, Zeller U, Baker JC, Grutzner F and Kaessmann H: The evolution of lncRNA repertoires and expression patterns in tetrapods. Nature 505: 635-640, 2014

7. Schmitt AM and Chang HY: Long noncoding RNAs in cancer pathways. Cancer Cell 29: 452-463, 2016.

8. Sun Z, Liu J, Chen C, Zhou Q, Yang S, Wang G, Song J, Li Z, Zhang Z, Xu J, et al: The biological effect and clinical application of long noncoding RNAs in colorectal cancer. Cell Physiol Biochem 46: 431-441, 2018.

9. Yang S, Sun Z, Zhou Q, Wang W, Wang G, Song J, Li Z, Zhang Z, Chang Y, Xia K, et al: MicroRNAs, long noncoding RNAs, and circular RNAs: Potential tumor biomarkers and targets for colorectal cancer. Cancer Manag Res 10: 2249-2257, 2018.

10. Ma T, Ma H, Zou Z, He X, Liu Y, Shuai Y, Xie M and Zhang Z: The long intergenic noncoding RNA 00707 promotes lung adenocarcinoma cell proliferation and migration by regulating Cdc42. Cell Physiol Biochem 45: 1566-1580, 2018.

11. Wang J, Luo Z, Yao T, Li W and Pu J: LINC00707 promotes hepatocellular carcinoma progression through activating ERK/JNK/AKT pathway signaling pathway. J Cell Physiol 234: 6908-6916, 2019.

12. Tu J, Zhao Z, Xu M, Chen M, Weng Q, Wang J and Ji J: LINC00707 contributes to hepatocellular carcinoma progression via sponging miR-206 to increase CDK14. J Cell Physiol 234: 10615-10624, 2019.

13. Xie M, Ma T, Xue J, Ma H, Sun M, Zhang Z, Liu M, Liu Y, $\mathrm{Ju} S$, Wang $\mathrm{Z}$ and De W: The long intergenic non-protein coding RNA 707 promotes proliferation and metastasis of gastric cancer by interacting with mRNA stabilizing protein HuR. Cancer Lett 443: 67-79, 2019.

14. Zhu H, He G, Wang Y, Hu Y, Zhang Z and Qian X: Long intergenic noncoding RNA 00707 promotes colorectal cancer cell proliferation and metastasis by sponging miR-206. Onco Targets Ther 12: 4331-4340, 2019.
15. Shao HJ, Li Q, Shi T, Zhang GZ and Shao F: LINC00707 promotes cell proliferation and invasion of colorectal cancer via miR-206/FMNL2 axis. Eur Rev Med Pharmacol Sci 23: 3749-3759, 2019.

16. Livak KJ and Schmittgen TD: Analysis of relative gene expression data using real-time quantitative PCR and the 2(-Delta Delta C(T)) method. Methods 25: 402-408, 2001.

17. Chai Y, Du Y, Zhang S, Xiao J, Luo Z, He F and Huang K MicroRNA-485-5p reduces O-GlcNAcylation of Bmi-1 and inhibits colorectal cancer proliferation. Exp Cell Res 368: 111-118, 2018

18. Lan Y, Xiao X, He Z, Luo Y, Wu C, Li L and Song X: Long noncoding RNA OCC-1 suppresses cell growth through destabilizing HuR protein in colorectal cancer. Nucleic Acids Res 46: 5809-5821, 2018.

19. Tsai KW, Lo YH, Liu H, Yeh CY, Chen YZ, Hsu CW, Chen WS and Wang JH: Linc00659, a long noncoding RNA, acts as novel oncogene in regulating cancer cell growth in colorectal cancer. Mol Cancer 17: 72, 2018.

20. Wang KC and Chang HY: Molecular mechanisms of long noncoding RNAs. Mol Cell 43: 904-914, 2011.

21. Dykes IM and Emanueli C: Transcriptional and post-transcriptional gene regulation by long non-coding RNA. Genomics Proteomics Bioinformatics 15: 177-186, 2017.

22. Fang Y and Fullwood MJ: Roles, functions, and mechanisms of long non-coding RNAs in cancer. Genomics Proteomics Bioinformatics 14: 42-54, 2016.

23. Chan JJ and Tay Y: Noncoding RNA: RNA regulatory networks in cancer. Int J Mol Sci 19: E1310, 2018.

24. Jia B, Wang Z, Sun X, Chen J, Zhao J and Qiu X: Long noncoding RNA LINC00707 sponges miR-370-3p to promote osteogenesis of human bone marrow-derived mesenchymal stem cells through upregulating WNT2B. Stem Cell Res Ther 10: 67, 2019.

25. Li T, Li Y and Sun H: MicroRNA-876 is sponged by long noncoding RNA LINC00707 and directly targets metadherin to inhibit breast cancer malignancy. Cancer Manag Res 11: 5255-5269, 2019

26. Zhu S, Zhou Z, Li Z, Shao J, Jiao G, Huang YE and Lin Y: Suppression of LINC00707 alleviates lipopolysaccharide-induced inflammation and apoptosis in PC-12 cells by regulated miR-30a-5p/Neurod 1. Biosci Biotechnol Biochem 83: 2049-2056, 2019.

27. Jing LL and Mo XM: Reduced miR-485-5p expression predicts poor prognosis in patients with gastric cancer. Eur Rev Med Pharmacol Sci 20: 1516-1520, 2016.

28. Mou X and Liu S: MiR-485 inhibits metastasis and EMT of lung adenocarcinoma by targeting Flot2. Biochem Biophys Res Commun 477: 521-526, 2016.

29. Wu J, Li J, Ren J and Zhang D: MicroRNA-485-5p represses melanoma cell invasion and proliferation by suppressing Frizzled7. Biomed Pharmacother 90: 303-310, 2017.

30. Lou C, Xiao M, Cheng S, Lu X, Jia S, Ren Y and Li Z: MiR-485-3p and miR-485-5p suppress breast cancer cell metastasis by inhibiting PGC-1 $\alpha$ expression. Cell Death Dis 7: e2159, 2016.

31. Hu XX, Xu XN, He BS, Sun HL, Xu T, Liu XX, Chen XX, Zeng KX, Wang SK and Pan YQ: microRNA-485-5p functions as a tumor suppressor in colorectal cancer cells by targeting CD147. J Cancer 9: 2603-2611, 2018. 